\title{
CRISPR-Cas9-mediated genomic multiloci integration in Pichia pastoris
}

\author{
Qi Liu ${ }^{1 \dagger}$, Xiaona Shi ${ }^{1 \dagger}$, Lili Song ${ }^{1}$, Haifeng Liu², Xiangshan Zhou ${ }^{1,2}$, Qiyao Wang ${ }^{1}$, Yuanxing Zhang ${ }^{1,3}$ \\ and Menghao Cai ${ }^{*}$ (D)
}

\begin{abstract}
Background: Pichia pastoris (syn. Komagataella phaffi) is a widely used generally recognized as safe host for heterologous expression of proteins in both industry and academia. Recently, it has been shown to be a potentially good chassis host for the production of high-value pharmaceuticals and chemicals. Nevertheless, limited availability of selective markers and low efficiency of homologous recombination make this process difficult and time-consuming, particularly in the case of multistep biosynthetic pathways. Therefore, it is crucial to develop an efficient and markerfree multiloci gene knock-in method in P. pastoris.
\end{abstract}

Results: A non-homologous-end-joining defective strain ( $\triangle k u 70$ ) was first constructed using the CRISPR-Cas9 based gene deficiency approach. It was then used as a parent strain for multiloci gene integration. Ten guide RNA (gRNA) targets were designed within $100 \mathrm{bp}$ upstream of the promoters or downstream of terminator, and then tested using an eGFP reporter and confirmed as suitable single-locus integration sites. Three high-efficiency gRNA targets ( $P_{A O X]}$ UP-g2, $P_{\text {TEF }}$ UP-g1, and $P_{F L D 1}$ UP-g1) were selected for double- and triple-locus co-integration. The integration efficiency ranged from 57.7 to $70 \%$ and 12.5 to $32.1 \%$ for double-locus and triple-locus integration, respectively. In addition, biosynthetic pathways of 6-methylsalicylic acid and 3-methylcatechol were successfully assembled using the developed method by one-step integration of functional genes. The desired products were obtained, which further established the effectiveness and applicability of the developed CRISPR-Cas9-mediated gene co-integration method in P. pastoris.

Conclusions: A CRISPR-Cas9-mediated multiloci gene integration method was developed with efficient gRNA targets in P. pastoris. Using this method, multiple gene cassettes can be simultaneously integrated into the genome without employing selective markers. The multiloci integration strategy is beneficial for pathway assembly of complicated pharmaceuticals and chemicals expressed in P. pastoris.

Keywords: Pichia pastoris, CRISPR-Cas9, Homology directed repair, Multiloci integration, Multistep enzymatic pathway

\section{Background}

Pichia pastoris (syn. Komagataella phaffii) is extensively used in the production of industrial enzymes and biopharmaceuticals owing to its notable advantages as a protein expression system [1,2]. Compared to other yeast species, $P$. pastoris has attracted a great deal of

\footnotetext{
*Correspondence: cmh022199@ecust.edu.cn

${ }^{+}$Qi Liu and Xiaona Shi are contributed equally as co-first authors ${ }^{1}$ State Key Laboratory of Bioreactor Engineering, East China University of Science and Technology, 130 Meilong Road, Shanghai 200237, China Full list of author information is available at the end of the article
}

attention, owing to its high secretion efficiency, reduced protein glycosylation, and high cell density cultivation $[3,4]$. Since $P$. pastoris is an FDA-approved generally recognized as safe host, it is widely used in the food and drug industry [5]. Recent studies have reported the heterologous synthesis of various pharmaceuticals and food additives such as polyketides [6-8], terpenoids [9, $10]$, and fatty acids $[11,12]$ using $P$. pastoris as a chassis host. Expression of these biopharmaceuticals and chemicals require re-construction and metabolic engineering of appropriate multistep enzymatic pathways. 
Although protein expression by episomal plasmids has been reported in P. pastoris $[13,14]$, it requires integration of heterologous genes into the genome for stable expression [15]. This is because homologous recombination is usually inefficient in P. pastoris, which is a non-conventional yeast, even with homologous flanking regions over several hundred base pairs [16]. In contrast, inclusion of fifty base pairs is generally sufficient to reach about $100 \%$ targeting efficiency in the conventional baker's yeast, i.e., Saccharomyces cerevisiae [17, 18]. Therefore, the assembly of multistep biosynthetic pathways for the expression of desired compounds in $P$. pastoris is associated with low integration efficiency, in addition to being a time-consuming process [19]. Moreover, availability of selection markers is limited in P. pastoris, which allows co-integration of genes into the genome by construction of mega plasmids bearing multiple expression cassettes [20,21], or involves laborintensive methods of introducing recycling makers [22, 23]. Therefore, it is crucial to develop an efficient, rapid, and marker-free gene integration approach for biosynthetic pathway assembly in P. pastoris.

Currently, CRISPR-Cas9 systems are widely used for gene editing, and these systems have been used for gene mutation, insertion, and deletion in various species [17, 18, 24-26]. Recently, Vogl and colleagues reported a CRISPR-Cas9 genome editing method in P. pastoris CBS7435 [27, 28]. In this study, the human codonoptimized cas gene and ribozyme-mediated guide RNA (gRNA) cassettes were placed on the same plasmid containing the ARS sequence, leading to effective gene mutation, deletion, and replacement [27]. In addition, $K U 70$, a key gene responsible for the non-homologousend-joining (NHEJ) repair mechanism, was knocked out. The double strand break (DSB) introduced by Cas9 cleavage was effectively repaired by the donor DNA containing genomic homologous sequence to prevent cell death in $\Delta k u 70$ strain [29]. This enhanced the homology directed repair (HDR) efficiency of gene deletion and replacement [28]. These reports demonstrated the suitability of marker-free donor DNA fragments in facilitating marker-free gene integration in $P$. pastoris, with the loss of plasmids containing Cas 9 and gRNA by continuous streaking $[27,30]$.

Integrating multiple genes in a single step will certainly facilitate pathway assembly involving various enzymes. Presently, gene expression cassettes are mainly loaded by a single plasmid for simultaneous integration of multiple genes in P. pastoris [31, 32]. Moreover, some general-purpose vectors and kits such as GoldenPiCS have been developed for the rapid assembly of multiple genes [20]. However, it is necessary to remove specific restriction enzyme sites on the gene of interest, promoter, and terminator, which ensures that the constructed plasmids can be properly linearized for the next round of plasmid construction or for transformation to competent cells [20]. Nevertheless, this step increases the difficulty in plasmid design since repeated rounds of plasmid construction are required [8]. The emergence of CRISPR-Cas9 gene editing system provides novel methods for the onestep genomic multiloci integration of various genes in P. pastoris. Expression of different gRNAs leads to DSBs at multiple sites in the genome simultaneously $[33,34]$. These cells can then be repaired by simultaneous integration of DNA fragments at the desired loci in the genome [35]. Additionally, the integration sites can strongly affect knock-in and expression efficiency of foreign genes [36]. To ensure integrity of the endogenous gene expression cassette, the homologous arms can be selected within promoters or terminators for single-crossover gene integration [4]. Recently, CRISPR-Cas9-mediated simultaneous integration of multiple genes has been achieved in various microbial chassis hosts including S. cerevisiae [35], Ogataea polymorpha [37], Kluyveromyces lactis [38], Escherichia coli [39], Aspergillus oryzae [40], and Penicillium chrysogenum [41], etc. However, genomic multiloci integration by CRISPR-Cas9 in P. pastoris has not yet been reported.

The present study aimed to develop a CRISPR-Cas9mediated genomic multiloci integration method in P. pastoris. To this end, the gRNA targets were first selected and tested within $100 \mathrm{bp}$ range of upstream of the identified promoter and downstream of the identified terminator. Using high-efficiency sites, we established a method for genomic double- (DLI) and triple-locus integration (TLI) in P. pastoris through a single step. Biosynthetic pathways of 6-methylsalicylic acid and 3-methylcatechol were then assembled by DLI and TLI, respectively. This genomic multiloci integration approach provides a simple, rapid, and convenient tool for co-expression of multiple proteins and assembly of complicated biosynthetic pathways in P. pastoris.

\section{Results \\ Integration efficiency of single locus varies with Cas9 cleavage site and gRNA}

The DSB caused by Cas9 is preferably repaired through NHEJ in P. pastoris. It has been reported that repressing NHEJ is an effective way to enhance HDR [29]. Accordingly, a KU70 deletion strain $(\Delta k u 70)$ was first constructed using CRISPR-Cas9 in the histidine-auxotroph P. pastoris GS115. The $\Delta k u 70$ strain grew normally on the carbon source of glucose, ethanol, and methanol (Additional file 1: Fig. S1). Therefore, it was used as a 
parental strain for subsequent gene integration experiments. Theoretically, the DSB from Cas9 cleavage was predominantly repaired by HDR to prevent cell death in $\Delta k u 70$ (Fig. 1a).

To achieve stable expression of exogenous genes, it is important to detect suitable integration sites that do not affect cell growth and metabolism of the parental strain after gene insertion. We then selected integration sites upstream of the identified promoters and downstream of an identified terminator in $P$. pastoris. Using this method, cells can maintain intact genomic endogenous expression cassettes, leading to protection from damage associated with DNA recombination. The gRNA targets were selected within 100-bp upstream of TEF1- $\alpha$ (PAS_FragB_0052), FLD1 (PAS_chr3_1028), AOX1 (PAS_ chr4_0821), and GAP (PAS_chr2-1_0437) promoter, and downstream of $A O X 1$ terminator by navigating the $5^{\prime}$-NGG-3' protospacer adjacent motif (PAM) sequence. Potential gRNA binding sequences were assessed with CHOPCHOP, a widely used web tool for CRISPR-based genome editing [42], and sequences with high scores were selected as candidate gRNA targets. In total, we selected 10 gRNA targets from 4 chromosomes of the host strain (Fig. 1b). The sequence and position of all gRNA targets are listed in Table 1. Subsequently, the donor DNA with enhanced green fluorescent protein (eGFP) cassette flanked by 1000-bp homologous arms was prepared as a
Table 1 Variable gRNA targets used in this study

\begin{tabular}{|c|c|c|}
\hline Name & Location & Sequence $\left(5^{\prime} \rightarrow 3^{\prime}\right)$ \\
\hline$P_{T E F /} \cup P-g 1$ & Chromosome 1 & GCAAGATGGTTAAAAGGTGA \\
\hline$P_{T E F 1} \cup P-g 2$ & & GAATGGGCAAGATGGTTAAA \\
\hline$P_{G A P} \cup P-g 1$ & Chromosome 2 & ATCGATAATAGTCGCATGTG \\
\hline$P_{G A P} \cup P-g 2$ & & TTTTAAGATTTCAATCTTGA \\
\hline$P_{F L D 1} \cup P-g 1$ & Chromosome 3 & GCGGCAGTAATTGATATCGT \\
\hline$P_{F L D 1} \cup P-g 2$ & & AGTAATTGATATCGTAGGGT \\
\hline$P_{A O X 1} \cup P-g 1$ & Chromosome 4 & AATCCAAATGTCATCATTGT \\
\hline$P_{A O X 1} \cup P-g 2$ & & GCGCCTACAATGATGACATT \\
\hline$P_{A O X I} \cup P-g 3$ & & TGGATTTGGTTGACTCATGT \\
\hline AOXTTDOWN & & TGACGCTTATTATACCCTTT \\
\hline
\end{tabular}

reporter for integration efficiency. $\mathrm{P}_{G A P}$ and $A O X T T$ were used as promoter and terminator of the eGFP coding gene, respectively. The homologous arms were selected as the upstream and downstream sequence of Cas9 cleavage site which is located 3-bp upstream of the PAM sequence. Colonies grown on plates after transformation were transferred into YND medium for further cultivation. The transformants with normal growth were collected to analyze eGFP fluorescence, which is expressed as relative fluorescence units (RFU) per unit of optical density measured at $600 \mathrm{~nm}\left(\mathrm{RFU} / \mathrm{OD}_{600}\right)$. Next, the integration efficiencies of gRNA targets were calculated (Additional

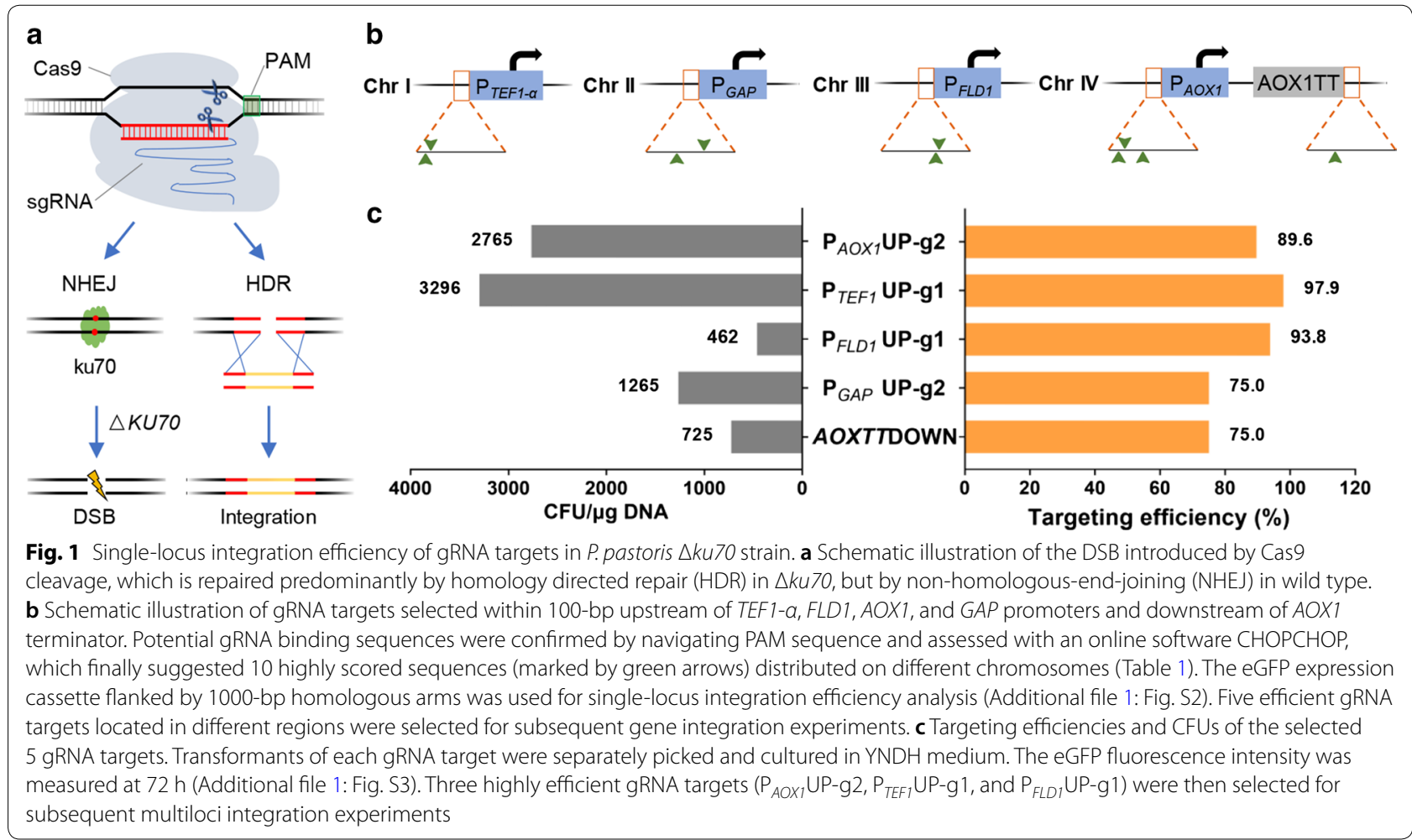


file 1: Fig. S2). gRNA targets in the same 100-bp region of a specific promoter or terminator cannot be used simultaneously due to the influence of the 1000-bp homologous arms. Therefore, we selected 5 gRNA targets, namely, $\mathrm{P}_{A O X 1}$ UP-g2, $\mathrm{P}_{T E F 1} \mathrm{UP}$-g1, $\mathrm{P}_{F L D 1} \mathrm{UP}-\mathrm{g} 1, \mathrm{P}_{G A P} \mathrm{UP}-\mathrm{g} 2$, and $A O X T T D O W N$, for the subsequent multiple-gene integration experiments. In the host strain of histidine-auxotroph GS115, HIS4 was used as a selective marker for the plasmids with gRNA and Cas9 expression cassettes. Since colonies need to be cultured in non-selective medium to promote plasmid loss [14], integration efficiency of the 5 selected gRNA targets was further analyzed in histidinecontaining YND (YNDH) medium, which could accelerate the loss of the episomal plasmid carrying the histidine coding gene, gRNA, and Cas9 expression cassettes. For each gRNA target, 96 colonies were selected and cultured for $72 \mathrm{~h}$ in $\mathrm{YNDH}$ medium.

Fluorescence intensity of transformants for a specific gRNA target is variable, possibly due to clonal variation. This is similar to previous reports on single-crossover integration in $P$. pastoris [36]. Some transformants with distinctly weak or strong fluorescence appeared at certain integration sites (Additional file 1: Fig. S3). Gene non-integration, ectopic integration, or multicopy integration may occur in these transformants because of the preferred nonhomologous recombination in P. pastoris [43]. Hence, these samples were not considered to be correctly integrated transformants. According to previous reports, virtually all surviving transformants in $\Delta k u 70$ strain showed correct integration of donor DNA [28]. Moreover, the transformants with medium intensity of GFP fluorescence were almost all GFP single-copy strains [36, 43], which were defined as correctly integrated strains. Analyzing the fluorescence intensity distribution of the correctly integrated strains revealed that fluorescence intensity fluctuated across a certain range for various colonies with eGFP cassette integrated at the same locus (Additional file 1: Fig. S3). The fluctuation range of fluorescence intensity and average fluorescence intensity of the colonies for each gRNA target is different, indicating that the integration locus has an impact on gene expression (Additional file 1: Fig. S3). The integration efficiency of each gRNA target was calculated as the ratio of the number of correctly integrated transformants to the total number of transformants. Our data showed high integration efficiency of all the five targets, i.e., $\mathrm{P}_{A O X 1} \mathrm{UP}-\mathrm{g} 2$ (89.6\%), $\mathrm{P}_{T E F 1} \mathrm{UP}$-g1 (97.9\%), $\mathrm{P}_{F L D 1} \mathrm{UP}-\mathrm{g} 1$ (93.8\%), $\mathrm{P}_{G A P} \mathrm{UP}-\mathrm{g} 2$ (75.0\%), and $A O X-$ TTDOWN (75.0\%) (Fig. 1c).

\section{Multiloci integration by CRISPR-Cas9 allows rapid knock-in of reporter genes}

Genome engineering methods for one-step multiplegene integration are favorable for the assembly of complex biochemical pathway. Here, as a proof-of-principle, we tested the possibility of multiloci integration of three reporter genes encoding fluorescent proteins eGFP (green), mCherry (red), and BFP (blue) (Fig. 2a). As mentioned above, three efficient gRNA targets of $\mathrm{P}_{A O X 1} \mathrm{UP}$-g2, $\mathrm{P}_{T E F 1} \mathrm{UP}-\mathrm{g} 1$, and $\mathrm{P}_{F L D 1} \mathrm{UP}-\mathrm{g} 1$ located on different chromosomes were used for these experiments. Multiple gRNA cassettes were assembled using the ribozymegRNA-ribozyme (RGR) operon regulated by the same promoter (Fig. 2a).

First, eGFP and mCherry were used to test the efficiency of DLI. Similar to single-locus integration, the transformants with distinctly weak or strong fluorescence were excluded from further analysis (Additional file 1: Fig. S4). As shown in Fig. 2b, the simultaneous integration efficiency of eGFP_P ${ }_{T E F 1} \mathrm{UP}$-g1 + mCherry_P $\mathrm{P}_{A O X 1} \mathrm{UP}$-g2 (eGFP_T + mCherry_A), eGFP_P ${ }_{A O X 1}$ UP-g $2+$ mCherry $\mathrm{P}_{\text {TEF1 }} \mathrm{UP}$-g1 (eGFP_A + mCherry_T), eGFP_P $\mathrm{P}_{T E F 1} \mathrm{UP}$ g1 + mCherry_P ${ }_{F L D 1}$ UP-g1 (eGFP_T + mCherry_F), and eGFP_P ${ }_{F L D 1} \mathrm{UP}-\mathrm{g} 1+\mathrm{mCherry} \mathrm{P}_{T E F 1} \mathrm{UP}-\mathrm{g} 1$ (eGFP_F + mCherry_T) was calculated to be $64.3 \%$, $62.5 \%, 57.7 \%$, and $70.0 \%$, respectively (Fig. $2 \mathrm{~b}$ ). These results indicated that insertion of two genes into the $P$. pastoris genome by this one-step integration method was feasible and effective. Notably, construction of the plasmid carrying gRNAs for both $\mathrm{P}_{A O X 1} \mathrm{UP}$-g2 and $\mathrm{P}_{F L D 1} \mathrm{UP}$-g1 was not successful, and therefore, we did not evaluate the combination of eGFP_A + mCherry_F and eGFP_F + mCherry_A. In addition, colony forming units (CFUs) were counted to analyze cell growth (Fig. 2b). Compared with the single-locus integration, the number of transformants obtained by DLI was markedly reduced (Figs. 1c, 2b).

Next, these three fluorescent proteins were used as reporters for evaluation of one-step TLI. We tested the following six different combinations of the three fluorescent proteins encoded by genes targeted to three distinct gRNA sites: eGFP_T + mCherry_A + BFP_F, eGFP_T + mCherry_F + BFP_A, eGFP_A + mCherry_T + BFP_F, eGFP_A + mCherry_F + BFP_T, eGFP_F + mCherry_T + BFP_A, and eGFP_F + mCherry_A + BFP_T (Fig. 2c, Additional file 1: Fig. S5). The TLI efficiency achieved ranged from 12.5 to $32.1 \%$ (Fig. 2c), which demonstrates the feasibility of TLI by CRISPR-Cas9 in P. pastoris. The differences in efficiency of the various combinations may be ascribed to variations in integration efficiency of specific genes at the same locus. Additionally, the number of transformants obtained by TLI (Fig. 2c) was markedly reduced compared with that obtained by DLI (Fig. 2b). This is probably due to the difficulty in repairing genomic DNA caused by increased DNA cleavages in the genome. The positive strain with eGFP, mCherry, and BFP was observed using a fluorescence microscope (Additional file 1: Fig. S6). 
a

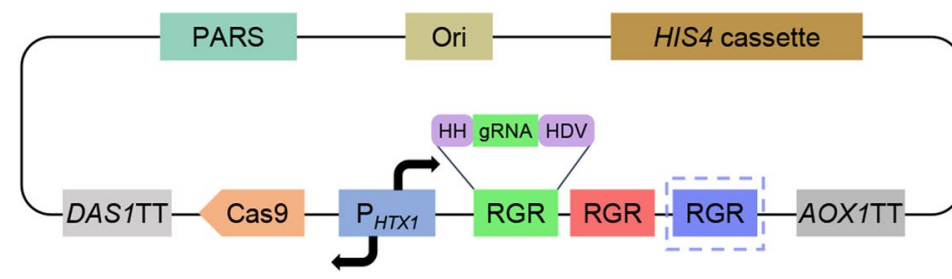

Double-locus integration
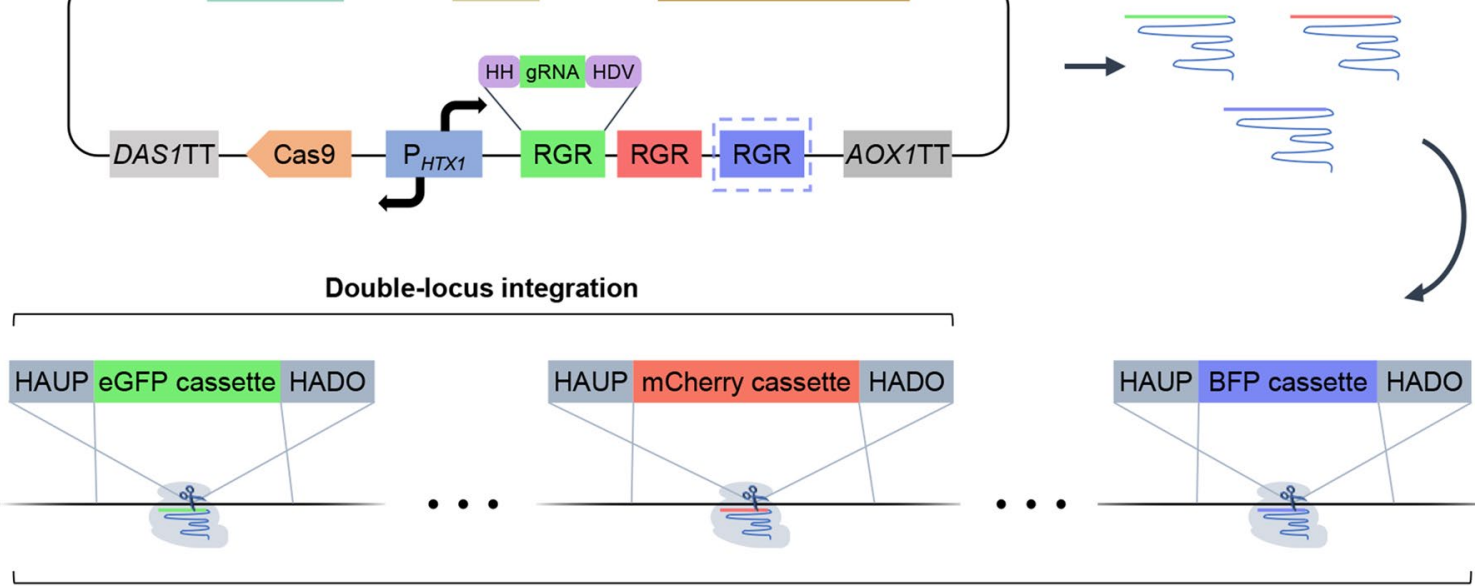

Triple-locus integration

b

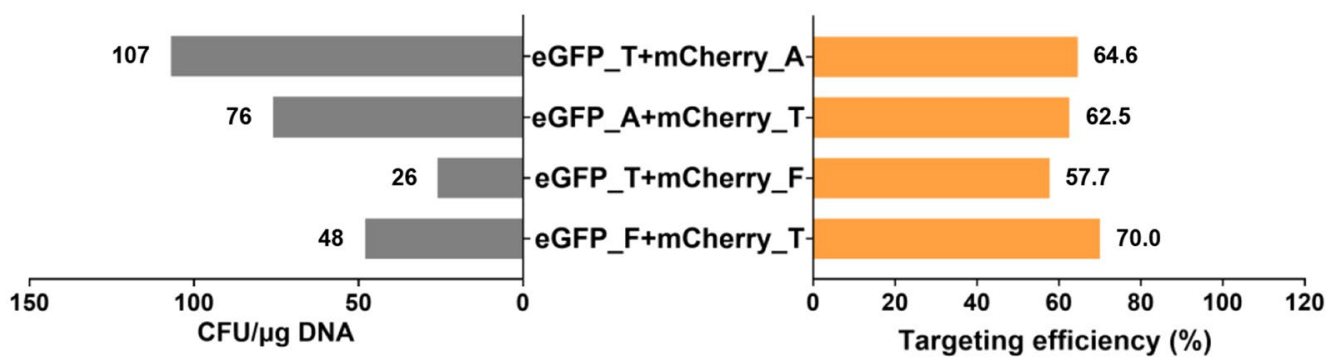

C

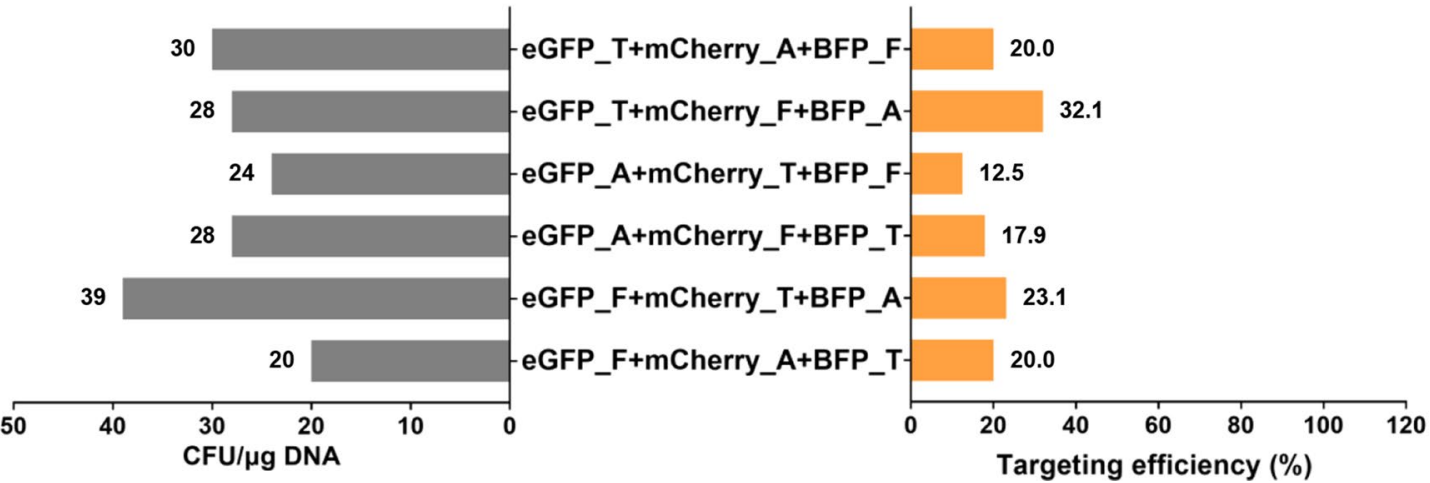

Fig. 2 CRISPR-Cas9-mediated multiloci integration in P. pastoris $\triangle k u 70$ strain. a Overview of double- and triple-locus integration. Three fluorescent proteins were used as reporter for efficiency analysis of double-locus (DLI, eGFP and mCherry) and triple-locus (TLI, eGFP, mCherry and BFP) integration. The gRNA coding sequence and its self-cleaving ribozymes flanking both sides constitute the RGR operon. Multiple tandem RGR operon and codon optimized CAS gene were co-regulated by the bidirectional promoter $P_{\text {HTXI }}$. The RGR operon shown in the dashed box represents TLI. b Targeting efficiencies and CFUs of DLI. Similar to single-locus integration experiments, the transformants with abnormal fluorescence intensity were excluded from analysis (Additional file 1: Fig. S4). The integration efficiency of the four experimental groups ranged

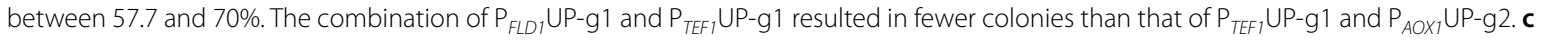
Targeting efficiencies and CFUs of TLI. As with previous experiments, only transformants that normally expressed three fluorescent proteins were counted (Additional file 1: Fig. S5). The CFUs and integration efficiency of TLI were relatively low for all six experimental groups independent of the donor DNAs

Further, we tested one-step integration of multicopy genes at certain gRNA targets, which would benefit the construction of high expression strains. egfp was used as a reporter gene, and DLI and TLI were effectively carried out. The genotypes of all transformants were verified by PCR to determine the integrated gRNA targeting sites 
(Additional file 1: Fig. S7). The proportion of double-copy strains was $79.2 \%$ in DLI at $\mathrm{P}_{A O X I} \mathrm{UP}-\mathrm{g} 2$ and $\mathrm{P}_{T E F 1} \mathrm{UP}-\mathrm{g} 1$. Moreover, the ratio of triple-copy strains was approximately $25 \%$ by TLI at $\mathrm{P}_{A O X 1} \mathrm{UP}-\mathrm{g} 2, \mathrm{P}_{T E F I} \mathrm{UP}-\mathrm{g} 1$, and $\mathrm{P}_{F L D 1} \mathrm{UP}$-g1. These results show that multiloci integration is effective for the construction of high-copy $P$. pastoris strains.

\section{Feasibility in multiloci integrative pathway building}

For further characterizing our method, we tested the use of DLI and TLI for multiloci integrative pathway building. In our previous study, we successfully constructed a biosynthetic pathway of terreic acid in P. pastoris [7]. In this pathway, an intermediate product 6-methylsalicylic acid (6-MSA) is synthesized by a polyketide synthase AtX and phosphopantetheinyl transferase NpgA (Fig. 3a). This compound can be further transformed to 3-methylcatechol by a salicylate 1-monooxygenase AtA (Fig. 3a). Therefore, 6-MSA and 3-methylcatechol can be utilized to assess the feasibility of multiloci integrative pathway assembly. As shown in Fig. 3b, the 6-MSA biosynthetic pathway was constructed by integrating at $X \_\mathrm{P}_{T E F 1} \mathrm{UP}-\mathrm{g} 1$ (at $\left.X \_\mathrm{T}\right)+n p g A_{-} \mathrm{P}_{A O X 1} \mathrm{UP}-\mathrm{g} 2$ (npgA_A), and the 3-methylcatechol biosynthetic pathway was constructed by integrating at $X \_\mathrm{T}+n p g A_{-} \mathrm{A}+a t A_{-} \mathrm{P}_{F L D 1} \mathrm{UP}-$ g1 (atA_F).

The efficiency for co-integration of atX and $n p g A$ was found to be approximately $65.9 \%$ after genotype verification by PCR (Fig. 3c, Additional file 1: Fig. S8). This was consistent with the DLI efficiency of mCherry and eGFP at atX_T + npgA_A (Fig. 2b). Next, the positive transformants were streaked on YPD plates to lose the plasmid carrying Cas9 and gRNAs, resulting in the final marker-free 6-MSA expression strains (Fig. 3d). Three recombinant strains of $\mathrm{K}-\mathrm{XN}$ were randomly selected for shake-flask culture and their extracts were analyzed by high-performance liquid chromatography (HPLC). All the tested strains produced 6-MSA successfully, which establishes the feasibility of this method in constructing the 6-MSA biosynthetic pathway (Additional file 1: Fig. S9).

Previous studies have shown that salicylate 1-monooxygenase encoded by atA catalyzes decarboxylative hydroxylation of 6-MSA to 3-methylcatechol [7]. Accordingly, the following three genes: atX, npgA, and $a t A$ were integrated into the genome of $P$. pastoris to further explore the feasibility of the TLI strategy (Fig. 3b). The integration efficiency was $23.5 \%$ in $a t X \_\mathrm{T}+n p g A_{-} \mathrm{A}+a t A_{-} \mathrm{F}$ (Fig. 3c, Additional file 1: Fig. S8), which is considerable to different expression patterns of the three fluorescence proteins (Fig. 2c). The marker-free 3-methylcatechol expression strains were finally obtained (Fig. 3d), and all the four tested strains produced 3-methylcatechol successfully. This further demonstrated the effectiveness of this co-integration strategy in constructing the 3-methylcatechol biosynthetic pathway (Additional file 1: Fig. S9).

Generally, recombinant strains capable of synthesizing 6-MSA and 3-methylcatechol have been obtained through one round of plasmid construction and strain screening by CRISPR-Cas9 mediated multiloci integration strategy, which greatly escalates the construction process of expression strains. Moreover, the recombinant strains obtained by DLI and TLI efficiently synthesized the desired products, which verifies that this gene integration method could be potentially valuable in both industry and academia.

\section{Discussion}

Recently, many studies have assembled multistep enzymatic pathways in P. pastoris for the heterologous synthesis of natural metabolites and pharmaceuticals. This makes it necessary to develop efficient methods for integration of multiple genes into the $P$. pastoris genome. Currently, CRISPR-Cas9-assisted simultaneous multiloci integration has been established in various species, such as S. cerevisiae [35], O. polymorpha [37], K. lactis [38], E. coli [39], A. oryzae [40], and P. chrysogenum [41], etc. This study reports a novel CRISPR-Cas9-mediated simultaneous multiloci integration method in P. pastoris, which will certainly facilitate the assembly of complex pathways in this host.

CRISPR-Cas9 system has been developed as a powerful tool for gene editing in a broad range of organisms [44]. In contrast to other yeast species, the development of CRISPR-Cas9 gene editing tools in P. pastoris has been more difficult. Using numerous experiments, Vogl and colleagues have explored some CRISPR-Cas9 tools which achieved gene mutation, deletion, and replacement in P. pastoris $[27,28]$. Here, multiloci integration of genes was conducted using CRISPR-Cas9 tools, which are an important supplement for the available genetic engineering toolboxes in P. pastoris. Previously, iterative rounds of plasmid construction or strain screening using selective markers were required for multiple genes integration in $P$. pastoris $[2,8,12]$. Our CRISPR-Cas9-mediated marker-free multiloci integration, therefore provides an alternative, efficient, and rapid approach for genetic engineering in P. pastoris. With 1000-bp homologous arms, DLI and TLI efficiency in P. pastoris ranged from 57.7 to $70 \%$ and 12.5 to $32.5 \%$, respectively. These efficiencies are similar to that of O. polymorpha $(30.6 \pm 2.4 \%$ in TLI with 1000-bp homologous arms [37]) but much lower than that in S. cerevisiae (58\% in DLI and 30.6\% in TLI with 50-bp homologous arms [45]; 84\% in TLI with 500 bp homologous arms [35]). These observations also 
a

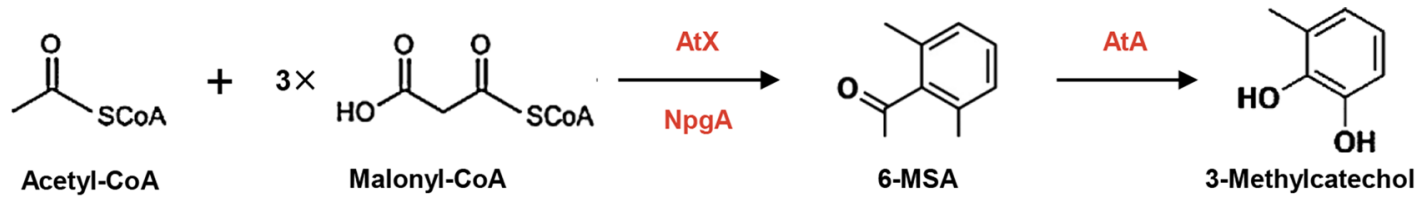

b

6-MSA

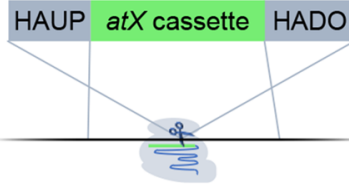

$P_{\text {TEF } 1}$ UP-g1

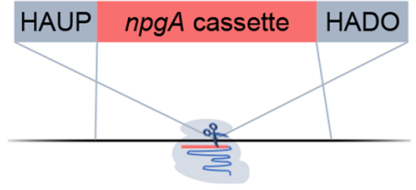

$\mathbf{P}_{\text {AOX1 }}$ UP-g2

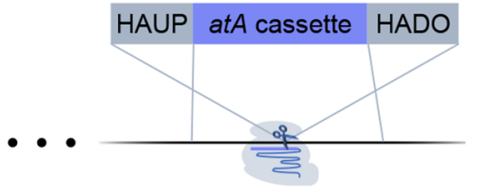

$P_{F L D 1}$ UP-g2

3-Methylcatechol

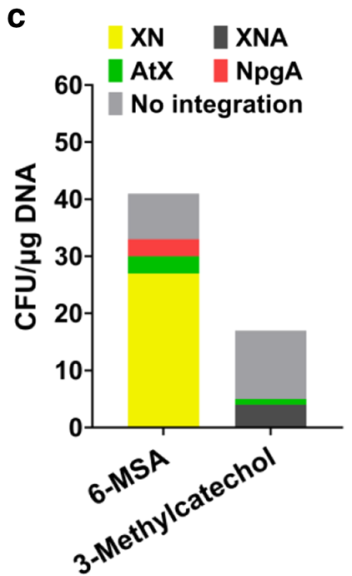

d

$\triangle k u 70$

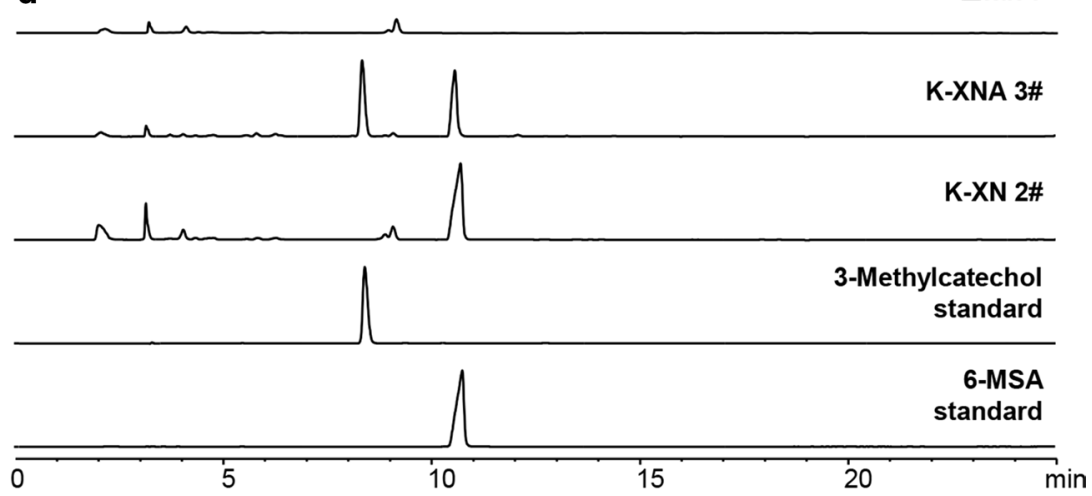

Fig. 3 CRISPR-Cas9-mediated multiloci integration of 6-MSA and 3-methylcatechol biosynthetic genes. a The biosynthetic pathway for 6-MSA and 3-methylcatechol. 6-MSA can be synthesized from acetyl-CoA and malonyl-CoA by a polyketide synthase AtX and a phosphopantetheinyl transferase NpgA. 3-methylcatechol can be synthesized from 6-MSA by a salicylate 1-monooxygenase AtA. $\mathbf{b}$ Overview of pathway assembly for production of 6-MSA and 3-methylcatechol in $\triangle k u 70$ strain. The $a t X$ and $n p g A$ expression cassettes were simultaneously integrated at $P_{\text {TEF }} U P$ - 1 and $P_{A O X 1} \cup P-g 2$, respectively. The $a t X, n p g A$, and atA expression cassettes were simultaneously integrated at $P_{T E F I} \cup P-g 1, P_{A O X 1} \cup P-g 2$, and $P_{F L D 1} \cup P-g 1$, respectively, for production of 3-methylcatechol. c The CFUs and genotypes of the transformants obtained by multiloci integration. According to the genotypes identified by PCR (Additional file 1: Fig. S8), 27 correct strains (K-XN) among 41 transformants in DLI and 4 correct strains (K-XNA) among 17 transformants in TLI. d HPLC analysis of organic extracts from culture broth. The strain K-XN 2\# integrating the atX and npgA expression cassettes and the strain K-XNA 3\# integrating the atX, npgA, and atA expression cassettes were cultured in YPD medium for $72 \mathrm{~h}$. Samples extracted from culture broth were analyzed for UV absorbance at $254 \mathrm{~nm}$. The HPLC analysis of other correct strains are shown in Additional file 1: Fig. S9

prove that homology directed repair is more difficult in $P$. pastoris than in S. cerevisiae.

Although only 5 gRNA targets were selected for further analysis and 3 were used for multiloci integration (Fig. 2d), the other targets also demonstrated good efficiency (Additional file 1: Table S1) and could be used for single-locus and multiloci integrations. The lower integration efficiency of the gRNA targets upstream of $\mathrm{P}_{G A P}$ and downstream of AOXTT may be attributed to the fact that the eGFP cassette and its homologous arms are flanked by $\mathrm{P}_{G A P}$ or $A O X T T$, thereby influencing donor DNA stability [30]. When two or three sites are simultaneously targeted in the $\Delta k u 70$ strain, cells with unrepaired DSB at any site may die because of its lethality. This may be the reason for the decrease of CFUs observed in multiloci integration (Figs. 1c, 2b, c), and especially, minimal CFUs seen for TLI (Fig. 2c). Additionally, the CFU level for $\mathrm{P}_{F L D I} \mathrm{UP}$-g1 was low in singlelocus integration and the combination of $\mathrm{P}_{F L D 1} \mathrm{UP}-\mathrm{g} 1$ and $\mathrm{P}_{T E F 1} \mathrm{UP}-\mathrm{g} 1$ produced fewer colonies compared to that of $\mathrm{P}_{A O X 1}$ UP-g1 and $\mathrm{P}_{T E F 1}$ UP-g1 (Fig. 2b). Thus, DSB at $\mathrm{P}_{F L D 1} \mathrm{UP}-\mathrm{g} 1$ may be more difficult to repair. In addition 
to conventional integration sites upstream of promoter and downstream of terminator, non-essential genes can also serve as potential integration sites in $P$. pastoris [46]. gRNAs targeting these non-essential genes can be designed and tested when more sites are needed for complex pathway assembly. Although HDR efficiency was promoted by repressing NHEJ via KU70 knockout, the increase in efficiency is mainly ascribed to decreased CFU in transformants. Enhancement of HDR ability may be beneficial for producing more positive colonies, which could be achieved by overexpressing the key protein of HDR mechanism such as Rad51 and Rad52 [47, 48].

Multicopy strains are often constructed and screened for production enhancement of compounds and proteins $[49,50]$. However, the identical expression cassette may be lost or misplaced when multicopy integration is performed by single-crossover in P. pastoris [43]. The integration sites selected in this study can be used for construction of multicopy strains by transforming donor DNAs containing identical cassettes and different homologous arms. Fluorescence experiments showed that the integration sites reported in this study allow efficient multicopy integration (Additional file 1: Fig. S7). Interestingly, many characteristic sites located near ARS sequences and non-transcribed spacers of rDNA on the genome have been used as gRNA targets for multicopy integration in S. cerevisiae [51, 52]. Similar strategies can also be used in P. pastoris to screen multicopy strains combined with antibiotic concentration screening.

This study showed that a combination of three integration sites facilitated one-step integration of three DNA fragments, and successfully generated strains synthesizing 6-MSA and 3-methylcatechol through only one round of transformation. Combination of four or more integration sites can be assessed in the future, which will further simplify the construction of multistep enzymatic pathways. The integration method established in this study can be combined with the vectors provided by Invitrogen and GoldenPiCS kit developed by Gasser and colleagues [20] to accelerate pathway construction in $P$. pastoris. The CRISPR-Cas9 based gene-editing method can be potentially used as a high-performance method for gene integration in $P$. pastoris in the future, and this will further extend its use as a chassis host in the field of synthetic biology.

\section{Conclusions}

An efficient CRISPR-Cas9 mediated one-step multiloci gene integration method was established in the widely used $P$. pastoris expression host. Using this method, biosynthetic pathways of 6-MSA and 3-methylcatechol were successfully constructed in $P$. pastoris through a single round of construction and transformation. The multiloci integration rapidly assembled pathways without the requirement of selective markers, which may extend the use of $P$. pastoris for synthesizing complicated pharmaceuticals and chemicals.

\section{Methods \\ Molecular biology techniques}

All restriction enzymes were purchased from TaKaRa Biomedical Technology (Beijing) Co., Ltd. Primers used in this study were ordered from Suzhou Genewiz Biotech Co., Ltd., China (Additional file 2: Table S2). For PCR experiments, standard protocols were applied following PCR amplification kit (TaKaRa). DNA fragments separated in an agarose gel were extracted with the Universal DNA Purification Kit (TIANGEN). The assembly of multi-fragments was achieved by ClonExpress ${ }^{\mathrm{TM}}$ II One Step Cloning Kit or ClonExpress MultiS One Step Cloning Kit (Vazyme Biotech Co., Ltd., China). Plasmid DNA and yeast genomic DNA were obtained using TIANprep Rapid Mini Plasmid Kit (TIANGEN) and TIANamp Yeast DNA Kit (TIANGEN), respectively.

\section{Strains and plasmids}

The strains and plasmids used in this study were listed in Additional file 2: Table S3. P. pastoris GS115 (Invitrogen) was employed as the parent strain. E. coli TOP10 (Invitrogen) served as a storage host for the construction and propagation of plasmids. The plasmids used for the expression of the CRISPR-Cas9 constructs are based on vector pPIC3.5K (Invitrogen). The vector pUC18 (Invitrogen) were used for construction of plasmids with donor DNA.

\section{Culture and growth conditions}

Escherichia coli cells were cultured at $37{ }^{\circ} \mathrm{C}$ in the LuriaBertani (LB) medium (10 g/L tryptone, $5 \mathrm{~g} / \mathrm{L}$ yeast extract and $10 \mathrm{~g} / \mathrm{L} \mathrm{NaCl}$ ) supplemented with $100 \mu \mathrm{g} /$ $\mathrm{mL}$ ampicillin. P. pastoris was cultivated at $30{ }^{\circ} \mathrm{C}$ in YPD medium $(20 \mathrm{~g} / \mathrm{L}$ tryptone, $10 \mathrm{~g} / \mathrm{L}$ yeast extract and $10 \mathrm{~g} / \mathrm{L}$ glucose). Yeast strains were pre-grown in YPD medium to $\mathrm{OD}_{600}$ of 2.0-8.0. Then the cells were harvested by centrifugation at $5000 \mathrm{~g}$ for $5 \mathrm{~min}$ and washed two times with sterile water. The obtained cells were inoculated into YPD medium, YND medium $(13.4 \mathrm{~g} / \mathrm{L}$ YNB,10 g/L glucose) or YNDH medium (13.4 g/L YNB,10 g/L glucose and $50 \mathrm{mg} / \mathrm{L}$ histidine) at an initial $\mathrm{OD}_{600}$ of 1.0. During culture phase, $2 \%(\mathrm{w} / \mathrm{v})$ glucose was fed into culture broth every $24 \mathrm{~h}$. The cell cultures were collected at $72 \mathrm{~h}$ for product extraction and analysis. The wild-type $\Delta k u 70$ strain was used as a negative control when necessary. 


\section{Construction of gRNA-Cas9 plasmids and donor cassette plasmids}

The gRNA-Cas9 plasmid was consists of autonomously replicating sequence PARS, homo sapiens codon optimized CAS9 (HsCAS9), DAS1TT terminator, RNA polymerase II bidirectional promoter $\mathrm{P}_{H T X 1}$, gRNA flanked by $\mathrm{HH}$ and HDV ribozymes and AOXTT terminator [27]. The gRNA-Cas9 plasmids were constructed on basis of plasmid pPIC3.5K-KU70-gRNA1 (Additional file 3: Additional methods) by designing primers to change gRNA targets. Two DNA fragments were amplified from plasmid pPIC3.5 K-KU70-gRNA1 by using corresponding primer pairs, and then were assembled to generate a series of gRNA-Cas9 plasmids carrying different gRNA CDS. In order to get gRNA-Cas9 plasmids carrying multiple gRNAs, they were assembled by ribozyme-gRNAribozyme (RGR) operon driven by the same promoter. The DNA fragment containing $\mathrm{P}_{A O X 1} \mathrm{UP}-\mathrm{g} 2, \mathrm{P}_{T E F 1} \mathrm{UP}-\mathrm{g} 1$ was amplified from plasmid pPIC3.5K-PAOX1upgRNA2, pPIC3.5 K-PTEF1up-gRNA1, respectively. Then two DNA fragments were assembled, leading to plasmid 3.5k-PAg2 + PTg1. Besides, plasmid 3.5k-PFg1+PTg1 was constructed in a similar way. To get plasmid $3.5 \mathrm{k}-$ PFg1 + PAg2 + PTg1, $\mathrm{P}_{F L D}$ UP-g1 fragment amplified from plasmid pPIC3.5K-PFLDup-gRNA1 was assembled with the fragment amplified from plasmid 3.5k-PAg2 + PTg1.

The universal vector pDGG was created to quickly assemble different homologous flanking regions for testing the efficiency of different gRNA targets. An eGFP reporter gene cassette amplified from pP-GFP was cloned into pUC18 by linearizing it with SacI and $K p n I$, resulting in the plasmid pDGG. The upstream and downstream homologous arms $(\sim 1 \mathrm{~kb})$ of the Cas 9 cut site were amplified from P. pastoris GS115 genomic DNA and joined by overlap-extension PCRs using primers listed in Additional file 2: Table S2, which then were assembled into the XbalI/SalI site of pDGG to generate donor DNA plasmid. The donor DNA plasmids bearing mCherry, BFP expression cassette (amplified from plasmid pBAD33-mCherry, pGAPZ-BFP, respectively) were derived from the plasmid pDGG. Expression cassette of mCherry amplified from plasmid pBAD33-mCherry with primer pairs of mChy-F/3AOX1 was assembled into linearized pDGG derivatives (amplified with primer pairs of 3AOX1F/ pGAPDO-R), resulting in donor DNA plasmids bearing mCherry. Moreover, the donor DNA plasmids carrying BFP expression cassette were obtained by amplification with primer pairs of pGAPF/3AOX1, 3AOX1F/ pGAPR from plasmid pGAPZ-BFP, pDGG derivatives, respectively.

To construct expression plasmids carrying exogenous gene expression cassettes, backbone-cloning vector $\mathrm{pDAg} 2$,
pDTg1, and pDFg1 were constructed with assembly cloning of upstream and downstream homologous arms of $\mathrm{P}_{A O X 1} \mathrm{UP}$-g2, $\mathrm{P}_{\mathrm{TEF} 1} \mathrm{UP}$-g1, and $\mathrm{P}_{F L D 1} \mathrm{UP}$-g1 by amplifying with primers listed in Additional file 2: Table S2 and religating the pUC18 by linearizing it with $S a c \mathrm{I}$ and $\mathrm{Xba \textrm {I }}$. The $n p g A$, at $X$, and $a t A$ expression cassettes were amplified from plasmids of pPIC3.5K-GAP-npgA, pPIC3.5K-pGAPat $X$, and $\mathrm{pPICZB}-a t A$, respectively, and then assembled into the ApaI/XhoI site of corresponding backbone-cloning vector $\mathrm{pDTg} 1, \mathrm{pDAg} 2$, and $\mathrm{pDFg} 1$, resulting in expression plasmids of pDTg1-npgA, pDAg2-atX, and pDFg1-atA, respectively.

\section{Transformation and screening}

The donor DNA was acquired by PCR amplification or enzyme digestion. The derivatives of the universal vector pDGG were linearized with ApaI and SpeI to obtain the specific donor DNA. For single-locus integration, $100 \mathrm{ng}$ gRNA-Cas9 plasmid and $1 \mu \mathrm{g}$ donor DNA were co-transformed into $\Delta k u 70$ by electroporation. To simultaneously integrate multiple genes, $1 \mu \mathrm{g}$ of each donor DNA was used with $100 \mathrm{ng}$ gRNA-Cas9 plasmid. Cells were cultivated on YND plates for 5 days, then randomly picked into 96-deep well plates (96-DWPs) containing YNDH medium. After $72 \mathrm{~h}$, cells were verified by genotyping PCRs or fluorescence measurements to determine integration efficiency of each gRNA target. Genomic DNA was isolated as template of PCR using TIANamp Yeast DNA Kit (TIANGEN Cat. \# DP307-02). A Microplate Reader (Synergy 2, BioTek) was used to measure fluorescent intensity of reporter protein. The cultures were diluted about fivefold with deionized water for fluorescence measurements. The fluorescence of eGFP, mCherry, and BFP was measured using ex/em $485 \pm 20 / 528 \pm 20$ filters (gain setting, 60), ex/em $590 \pm 20 / 645 \pm 40$ filters (gain setting, 60), and ex/em $360 \pm 20 / 460 \pm 20$ filters (gain setting, 60), respectively. The fluorescence was normalized to the $\mathrm{OD}_{600}$ for data analysis.

\section{Product extraction and HPLC analysis}

Ten $\mathrm{mL}$ culture broth was fully extracted with the same volume of ethyl acetate. The organic phase was distilled under reduced pressure and dissolved in methanol. All samples were analyzed by high-performance liquid chromatography (Agilent Technologies 1260 series). It is equipped with a C18 reverse column (Kromasil ${ }^{\mathrm{TM}}$, Sweden, $250 \mathrm{~mm} \times 4.6 \mathrm{~mm} \times 5 \mu \mathrm{m}, 100 \AA$-spherical silica) with the following gradient: $0.1 \%$ acetate solution (A) versus $100 \%$ acetonitrile (B) (0 min, 25\% B; $20 \mathrm{~min}$, $65 \% \mathrm{~B} ; 22-25 \mathrm{~min}, 100 \% \mathrm{~B}$ ), running at a flow of $1 \mathrm{ml} /$ min, column temperature of $30{ }^{\circ} \mathrm{C}$ and absorption wavelength of $254 \mathrm{~nm}$. 


\section{Supplementary information}

Supplementary information accompanies this paper at https://doi. org/10.1186/s12934-019-1194-x.

Additional file 1. Additional figures and legends supporting the results described in text.

Additional file 2. Lists of strains, plasmids, primers and gRNAs.

Additional file 3. Description of methods mainly related with the additional files.

\section{Abbreviations}

GRAS: generally recognized as safe; NHEJ: non-homologous-end-joining; HDR homology directed repair; DSB: double strand break; PAM: protospacer adjacent motif; YNDH: histidine-containing YND medium; RGR: ribozyme-gRNAribozyme; DLI: double-locus integration; TLI: triple-locus integration; 6-MSA: 6-methylsalicylic acid; HPLC: high-performance liquid chromatography.

\section{Acknowledgements}

Not applicable.

\section{Authors' contributions}

MC and QL conceived the project. XS and QL performed the experiments. LS participated in strain construction. $\mathrm{HL}$ directed the product analysis. XS, QL and $\mathrm{MC}$ analyzed the results and prepared the manuscript. XZ, QW and YZ reviewed the manuscript. All authors read and approved the final manuscript.

\section{Funding}

This work was supported by the National Key R\&D Program of China (2018YFC1706200), National Natural Science Foundation of China (31870073), Fundamental Research Funds for the Shanghai Science and Technology Innovation Action Plan (17JC 1402400), Shanghai Rising-Star Program, China (19QA1402700), the 111 Project, China (B18022), Fundamental Research Funds for the Central Universities, China (22221818014), Research Program of State Key Laboratory of Bioreactor Engineering.

\section{Availability of data and materials}

The materials and datasets for the current study are available from the corresponding author on reasonable request.

\section{Ethics approval and consent to participate}

Not applicable.

\section{Consent for publication}

Not applicable.

\section{Competing interests}

The authors declare that they have no competing interests.

\section{Author details}

1 State Key Laboratory of Bioreactor Engineering, East China University of Science and Technology, 130 Meilong Road, Shanghai 200237, China. ${ }^{2}$ Chinare Resources Angde Biotech Pharmaceutical Co., Ltd., 78 E-jiao Street, Liaocheng, China. ${ }^{3}$ Shanghai Collaborative Innovation Center for Biomanufacturing, 130 Meilong Road, Shanghai 200237, China.

Received: 31 May 2019 Accepted: 14 August 2019

Published online: 21 August 2019

\section{References}

1. Safder I, Khan S, Islam I-U, Ali MK, Bibi Z, Waqas M. Pichia pastoris expression system: a potential candidate to express protein in industrial and biopharmaceutical domains. Biomed Lett. 2018;4:1-14.

2. Peña DA, Gasser B, Zanghellini J, Steiger MG, Mattanovich D. Metabolic engineering of Pichia pastoris. Metab Eng. 2018;50:2-15.
3. Yang Z, Zhang Z. Engineering strategies for enhanced production of protein and bio-products in Pichia pastoris: a review. Biotechnol Adv. 2017;36:182-95.

4. Schwarzhans JP, Luttermann T, Geier M, Kalinowski J, Friehs K. Towards systems metabolic engineering in Pichia pastoris. Biotechnol Adv. 2017;35:681-710

5. VoglT, Hartner FS, Glieder A. New opportunities by synthetic biology for biopharmaceutical production in Pichia pastoris. Curr Opin Biotechnol. 2013;24:1094-101.

6. Gao L, Cai M, Shen W, Xiao S, Zhou X, Zhang Y. Engineered fungal polyketide biosynthesis in Pichia pastoris: a potential excellent host for polyketide production. Microb Cell Fact. 2013;12:77.

7. Kong C, Huang H, Xue Y, Liu Y, Peng Q, Liu Q, Xu Q, Zhu Q, Yin Y, Zhou X, Zhang Y, Cai M. Heterologous pathway assembly reveals molecular steps of fungal terreic acid biosynthesis. Sci Rep. 2018;8:2116.

8. Liu Y, Bai C, Liu Q, Xu Q, Qian Z, Peng Q, Yu J, Xu M, Zhou X, Zhang Y, Cai M. Engineered ethanol-driven biosynthetic system for improving production of acetyl-CoA derived drugs in Crabtree-negative yeast. Metab Eng. 2019;54:275-84.

9. Araya-Garay JM, Feijoo-Siota L, Rosa-Dos-Santos F, Veiga-Crespo P, Villa TG. Construction of new Pichia pastoris X-33 strains for production of lycopene and $\beta$-carotene. Appl Microbiol Biotechnol. 2012;93:2483-92.

10. Zhao C, Gao X, Liu X, Wang Y, Yang S, Wang F, Ren Y. Enhancing biosynthesis of a ginsenoside precursor by self-assembly of two key enzymes in Pichia pastoris. J Agric Food Chem. 2016;64:3380-5.

11. Meesapyodsuk D, Chen Y, Ng SH, Chen J, Qiu X. Metabolic engineering of Pichia pastoris to produce ricinoleic acid, a hydroxy fatty acid of industrial importance. J Lipid Res. 2015;56:2102-9.

12. Kim SH, Roh KH, Kim K-S, Kim HU, Lee KR, Kang HC, Kim JB. Coexpression of multiple genes reconstitutes two pathways of very long-chain polyunsaturated fatty acid biosynthesis in Pichia pastoris. Biotechnol Lett. 2014;36:1843-51

13. Madsen CK, Vismans G, Brinch-Pedersen $H$. The PARS sequence increase the efficiency of stable Pichia pastoris transformation. J Microbiol Methods. 2016;129:1-7.

14. Camattari A, Goh A, Yip LY, Tan AHM, Ng SW, Tran A, Liu G, Liachko I, Dunham MJ, Rancati G. Characterization of a panARS-based episomal vector in the methylotrophic yeast Pichia pastoris for recombinant protein production and synthetic biology applications. Microb Cell Fact. 2016;15:139.

15. Löbs AK, Schwartz C, Wheeldon I. Genome and metabolic engineering in non-conventional yeasts: current advances and applications. Synth Syst Biotechnol. 2017;2:198-207.

16. Madhavan A, Jose AA, Binod P, Sindhu R, Sukumaran RK, Pandey A, Castro GE. Synthetic biology and metabolic engineering approaches and its impact on non-conventional yeast and biofuel production. Front Energy Res. 2017:5:8.

17. Raschmanova H, Weninger A, Glieder A, Kovar K, Vogl T. Implementing CRISPR-Cas technologies in conventional and non-conventional yeasts: current state and future prospects. Biotechnol Adv. 2018;36:641-65.

18. Cai P, Gao J, Zhou Y. CRISPR-mediated genome editing in non-conventional yeasts for biotechnological applications. Microb Cell Fact. 2019;18:63.

19. Kang Z, Huang H, Zhang Y, Du G, Chen J. Recent advances of molecular toolbox construction expand Pichia pastoris in synthetic biology applications. World J Microbiol Biotechnol. 2017;33:19.

20. Prielhofer R, Barrero JJ, Steuer S, Gassler T, Zahrl R, Baumann K, Sauer M, Mattanovich D, Gasser B, Marx H. GoldenPiCS: a Golden Gate-derived modular cloning system for applied synthetic biology in the yeast Pichia pastoris. BMC Syst Biol. 2017;11:123.

21. Liu Y, Tu X, Xu Q, Bai C, Kong C, Liu Q, Yu J, Peng Q, Zhou X, Zhang Y, Cai $M$. Engineered monoculture and co-culture of methylotrophic yeast for de novo production of monacolin J and lovastatin from methanol. Metab Eng. 2017;45:189-99.

22. Li C, Lin Y, Zheng $X$, Yuan Q, Pang N, Liao X, Huang Y, Zhang X, Liang $S$ Recycling of a selectable marker with a self-excisable plasmid in Pichia pastoris. Sci Rep. 2017;7:11113.

23. Jiao L, Zhou Q, Yan Y. Efficient gene disruption by posttransformational directed internal homologous recombination in Pichia pastoris. Anal Biochem. 2019;576:1-4 
24. Hsu PD, Lander ES, Feng Z. Development and applications of CRISPRCas9 for genome engineering. Cell. 2014;157:1262-78.

25. Sternberg S, Doudna J. Expanding the biologist's toolkit with CRISPRCas9. Mol Cell. 2015;58:568-74.

26. Komor AC, Badran AH, Liu DR. CRISPR-based technologies for the manipulation of eukaryotic genomes. Cell. 2017;168:20-36.

27. Weninger A, Hatzl AM, Schmid C, Vogl T, Glieder A. Combinatorial optimization of CRISPR/Cas9 expression enables precision genome engineering in the methylotrophic yeast Pichia pastoris. J Biotechnol. 2016:235:139-49.

28. Weninger A, Fischer JE, Raschmanová H, Kniely C, Vogl T, Glieder A. Expanding the CRISPR/Cas9 toolkit for Pichia pastoris with efficient donor integration and alternative resistance markers. J Cell Biochem. 2018;119:3183-98.

29. Näätsaari L, Mistlberger B, Ruth C, Hajek T, Hartner FS, Glieder A. Deletion of the Pichia pastoris KU70 homologue facilitates platform strain generation for gene expression and synthetic biology. PLoS ONE. 2012;7:e39720.

30. Gassler T, Heistinger L, Mattanovich D, Gasser B, Prielhofer R. CRISPR/ Cas9-mediated homology-directed genome editing in Pichia pastoris. In: Gasser B, Mattanovich D, editors. Recombinant protein production in yeast. New York: Springer; 2019. p. 211-25.

31. Obst U, Lu TK, Sieber V. A modular toolkit for generating Pichia pastoris secretion libraries. ACS Synth Biol. 2017;6:1016-25.

32. Schreiber C, Müller H, Birrenbach O, Klein M, Heerd D, Weidner T, Salzig D, Czermak P. A high-throughput expression screening platform to optimize the production of antimicrobial peptides. Microb Cell Fact. 2017;16:29.

33. Cong L, Ran FA, Cox D, Lin S, Barretto R, Habib N, Hsu PD, Wu X, Jiang W, Marraffini LA, Zhang F. Multiplex genome engineering using CRISPR/Cas systems. Science. 2013;339:819-23.

34. Zerbini F, Zanella I, Fraccascia D, König E, Irene C, Frattini LF, Tomasi M, Fantappiè L, Ganfini L, Caproni E, Parri M, Grandi A, Grandi G. Large scale validation of an efficient CRISPR/Cas-based multi gene editing protocol in Escherichia coli. Microb Cell Fact. 2017;16:68.

35. Ronda C, Maury J, Jakociunas T, Jacobsen SAB, Germann SM, Harrison SJ, Borodina I, Keasling JD, Jensen MK, Nielsen AT. CrEdit: CRISPR mediated multi-loci gene integration in Saccharomyces cerevisiae. Microb Cell Fact. 2015;14:97.

36. Vogl T, Gebbie L, Palfreyman RW, Speight R. Effect of plasmid design and type of integration event on recombinant protein expression in Pichia pastoris. Appl Environ Microbiol. 2018;84:e02712-7.

37. Wang L, Deng A, Zhang Y, Liu S, Liang Y, Bai H, Cui D, Qiu Q, Shang X, Yang Z, He X, Wen T. Efficient CRISPR-Cas9 mediated multiplex genome editing in yeasts. Biotechnol Biofuels. 2018;11:277.

38. Horwitz AA, Walter JM, Schubert MG, Kung SH, Hawkins K, Platt DM, Hernday AD, Mahatdejkul-Meadows T, Szeto W, Chandran SS, Newman JD. Efficient multiplexed integration of synergistic alleles and metabolic pathways in yeasts via CRISPR-Cas. Cell Syst. 2015;1:88-96.

39. Ronda C, Pedersen LE, et al. CRMAGE: CRISPR optimized mage recombineering. Sci Rep. 2016;6:19452
40. Katayama T, Nakamura $H$, Zhang $Y$, et al. Forced recycling of an AMA1based genome-editing plasmid allows for efficient multiple gene deletion/integration in the industrial filamentous fungus Aspergillus oryzae. Appl Environ Microbiol. 2019;85:e01896-18.

41. Pohl C, Kiel J, Driessen AJM, et al. CRISPR/Cas9 based genome editing of Penicillium chrysogenum. ACS Synth Biol. 2016;5:754-64.

42. Labun K, Montague TG, Gagnon JA, Thyme SB, Valen E. CHOPCHOP v2: a web tool for the next generation of CRISPR genome engineering. Nucleic Acids Res. 2016:44:W272-6.

43. Schwarzhans JP, Wibberg D, Winkler A, Luttermann T, Kalinowski J, Friehs $\mathrm{K}$. Integration event induced changes in recombinant protein productivity in Pichia pastoris discovered by whole genome sequencing and derived vector optimization. Microb Cell Fact. 2016;15:84.

44. Adiego-Pérez B, Randazzo P, Daran JM, Verwaal R, Roubos JA, DaranLapujade P, van der Oost J. Multiplex genome editing of microorganisms using CRISPR-Cas. FEMS Microbiol Lett. 2019;366:fnz086.

45. Jakočiūnas T, Sonde I, Herrgård M, Harrison SJ, Kristensen M, Pedersen LE, Jensen MK, Keasling JD. Multiplex metabolic pathway engineering using CRISPR/Cas9 in Saccharomyces cerevisiae. Metab Eng. 2015;28:213-22.

46. Zhu J, Gong R, Zhu Q, He Q, Xu N, Xu Y, Cai M, Zhou X, Zhang Y, Zhou M. Genome-wide determination of gene essentiality by transposon insertion sequencing in yeast Pichia pastoris. Sci Rep. 2018;8:10223.

47. Ceccaldi R, Rondinelli B, D'Andrea AD. Repair pathway choices and consequences at the double-strand break. Trends Cell Biol. 2016;26:52-64.

48. Shao S, Ren C, Liu Z, Bai Y, Chen Z, Wei Z, Wang X, Zhang Z, Xu K. Enhancing CRISPR/Cas9-mediated homology-directed repair in mammalian cells by expressing Saccharomyces cerevisiae Rad52. Int J Biochem Cell Biol. 2017;92:43-52.

49. Aw R, Polizzi KM. Liquid PTVA: a faster and cheaper alternative for generating multi-copy clones in Pichia pastoris. Microb Cell Fact. 2016;15:29.

50. Sallada ND, Harkins LE, Berger BW. Effect of gene copy number and chaperone coexpression on recombinant hydrophobin HFBI biosurfactant production in Pichia pastoris. Biotechnol Bioeng. 2019. https://doi. org/10.1002/bit.26982.

51. Apel AR, D'Espaux L, Wehrs M, Sachs D, Li RA, Tong GJ, Garber M, Nnadi O, Zhuang W, Hillson NJ, Keasling JD, Mukhopadhyay A. A Cas9-based toolkit to program gene expression in Saccharomyces cerevisiae. Nucleic Acids Res. 2017:45:496-508.

52. Moon HY, Lee DW, Sim GH, Kim HJ, Hwang JY, Kwon MG, Kang BK, Kim JM, Kang HA. A new set of rDNA-NTS-based multiple integrative cassettes for the development of antibiotic-marker-free recombinant yeasts. J Biotechnol. 2016:233:190-9.

\section{Publisher's Note}

Springer Nature remains neutral with regard to jurisdictional claims in published maps and institutional affiliations.
Ready to submit your research? Choose BMC and benefit from:

- fast, convenient online submission

- thorough peer review by experienced researchers in your field

- rapid publication on acceptance

- support for research data, including large and complex data types

- gold Open Access which fosters wider collaboration and increased citations

- maximum visibility for your research: over 100M website views per year

At BMC, research is always in progress.

Learn more biomedcentral.com/submissions 